\title{
Areca nut habits amongst children in Tower Hamlets, London
}

\author{
Prevalence, age of onset and demographic relationships of different areca nut habits amongst children in Tower \\ Hamlets, London by P. Farrand, R. M. Rowe, A. Johnston, and H. Murdoch Br Dent J 2001; 190: 150-154
}

\section{Objective}

To examine prevalence and demographic relationships of different areca nut habits amongst children.

\section{Design}

Self-administered questionnaire.

\section{Subjects}

Children aged between 11 and 15. Of 800 questionnaires distributed, 704 were fully completed $(88 \%)$.

\section{Setting}

Two secondary schools in the London district of Tower Hamlets.

\section{Measures}

Demographic, areca nut habits used, age first used, still using, frequency of use.

\section{Results}

Users of any areca nut habit were exclusively from the South Asian population. Of this population, $77 \%$ had engaged in a habit, and dependent upon habit between 54 and $92 \%$ of these still remained current users. The highest prevalence of current use for boys and girls respectively was for areca nut alone $(36 \%$, $43 \%)$, followed by mistee pan $(35 \%, 29 \%)$, betel-quid $(27 \%$, $26 \%)$ and pan masala $(14 \%, 16 \%)$. Of the current users, $44 \%$ engaged in one habit only, $24 \%$ two, $20 \%$ three and $13 \%$ all four. The highest period of risk for starting to use areca nut alone, betel-quid and mistee pan was between ages 5 and 12, whilst for pan masala it was after 10. Boys had a significantly higher risk of beginning use before $10(P<.001)$ and a higher frequency of use for pan masala $(P<.01)$, areca nut alone $(P<.05)$ and betel-quid $(P=.06)$ than girls. The frequency of using each habit was between 3 and 5 episodes per week, however boys use pan masala approximately 10 times per week.

\section{Conclusion}

South Asian children may already be experienced users of areca nut. Greater attention should be directed towards identifying signs of oral submucous fibrosis, oral cancer and other potentially malignant lesions within the South Asian population.

\section{Comment}

This paper reports the outcomes of a 1 questionnaire investigating self-reports of four variants of areca nut consumption in a large sample of 704, largely Bangladeshi, children aged 11-14 years. Areca nut consumption by itself is the most common habit, consumed by $36 \%$ of boys and $43 \%$ of girls respectively. The consumption frequency of all variants was low, ranging from three to five weekly episodes, with the exception of pan masala (a proprietary form of betel-quid or paan) which was consumed by boys 10 times per week.

The authors acknowledge that at these levels of consumption there appears little health risk of oral sub-mucous fibrosis from these items alone. The authors were not able to collect data about the addition of tobacco to these products. There has been a developing concern in the UK about the health compromising aspects of consumption of gutkha - which includes tobacco - by children from South Asian communities. ${ }^{1}$ This paper helps to contextualise these concerns. Thirty five per cent of boys and $29 \%$ of girls reported themselves as current chewers of mistee pan (commercially prepared areca nut 'sweets') which, the authors note, might also include gutkha.

The findings demonstrate the difficulty of understanding complex, traditional behaviours using self-complete questionnaires. Areca nut preparation and consumption in betel quid or paan has traditionally been according to individual preference. As this investigation reports, proprietary variants are now being developed, packaged and sold under a range of trade names. This leads, as the authors recognise, to variation in terminology and content.

Health promotion strategies are recommended. This might include health education within schools. These young consumers could be advised to read the labels of products to find out their contents. The value of this advice is questionable: inadequate labelling information and inappropriate composition of the proprietary variants has been identified. ${ }^{2}$ Regulations are available to address these manufacturing deficiencies. ${ }^{3,4}$

\section{Ray Croucher}

Senior Lecturer in Dental Public Health, St Bartholomew's and the Royal London School of Medicine and Dentistry

1 R Bedi. What is gutkha. British Dental Associa tion News 1999; 12(4): 20.

2. R. Croucher, M. O'Farrell. Community based Prevention of Oral Cancer: Health Promotion Strategies relating to betal quid chewing in East London. St Bartholomew's and the Royal London School of Medicine and Dentistry/ Community and Salaried Dental Services. Tower Hamlets Healthcare NHS Trust, 1998.

3. The Food Labelling Regulations, 1996.

4. The Tobacco Products Labelling (Safety) Regulations, 1991. 